\title{
Prediction of Severity of Delusion Based on Jumping-to- Conclusion Bias in Schizophrenia Patients
}

\author{
Zahra Saffarian ${ }^{1 *}$, Mohammad Ali Goodarzi², Maryam Abbasian³, Javad Molazadeh², Habib Hadianfard \\ 1. Department of Clinical Psychology, University of Social Welfare and Rehabilitation Sciences, Tehran, Iran. \\ 2. Department of Clinical Psychology, Faculty of Education and Psychology, Shiraz University, Shiraz, Iran. \\ 3. Department of Clinical Psychology, Sari Branch, Islamic Azad University of Sari, Sari, Iran.
}

Cftation: Saffarian Z, Goodarzi MA, Abbasian M, Molazadeh J, Hadianfard H. Prediction of severity of delusion based on jumping-to-conclusion bias in Schizophrenia patients. Iranian Rehabilitation Journal. 2016; 14(1): 69-73. http://dx.crossref.org/10.15412/J.IRJ.08140111

: http://dx.crossref.org/10.15412/J.IRJ.08140111

Article info:

Received: 05 Sep. 2015

Accepted: 28 Dec. 2015

\section{Keywords:}

Jumping-to-conclusion bias, Delusion, Similarity task

\section{A B S T RACT}

Objectives: New cognitive theories of delusions have proposed that deficit or bias in inference stage (a stage of normal belief formation) is significant in delusion formation. The aim of this study was predicting the severity of delusions based on jumping-to-conclusion bias in patients with schizophrenia.

Methods: The sample consisted of 60 deluded patients with schizophrenia who were selected from the Ebnesina and Razi hospitals in Shiraz using convenience sampling method. The Similarity Task was used to measure the jumping-to-conclusion biases.

Results: Its results have shown that the jumping-to-conclusion biases could predict a great part of the variance of delusions.

Discussion: These results generally indicated that the jumping to conclusion biases may provide a more useful explanation for the delusion formation.

\section{Introduction}

$\mathbf{T}$

he beginner viewpoints believed that delusions were different from normal belief [1]. The current cognitive researches have suggested that abnormality in the normal stage of development of belief plays an important role in the creation of delusion [2]. According to the new model in cognitive theories, all stages in creation of a new belief, including attention, inference, and search for rejection or confirmation of belief should be attend [2].
Some theories emphasis on abnormality in the stage of inference, one of which is 'jumping-to-conclusion bias' theory, which refers to a hasty decision based on limited evidence [3]. Garety (2006), claimed that the people who suffere from delusion have jumping-to-conclusion bias about their surrounding issues. This bias leads to the rapid acceptance of opinions in the case of limited evidence. In addition, these individuals have full assurance about their own judgment [4].

There are two hypotheses which have attempted to prove the origin of this bias [5] Although the origin of

* Corresponding Author:

Zahra Saffarian, PhD Candidate

Address: Department of Clinical Psychology, University of Social Welfare and Rehabilitation Sciences, Kodakyar Ave., Daneshjoo Blvd., Evin, Tehran, Iran Tel: +98 (917) 1255979

E-mail: saffarian.psy@gmail.com 
jumping-to-conclusion bias is not exactly understood [1, 2]. The first hypothesis states that the patients with delusion have tendency to over-estimate their answers as a more correct one at the beginning of the decision-making process $[6,7]$, and the second explicitly suggests that such patients have low decision thresholds; therefore, they use less information for making decision [8, 9]. Kapur claims that jumping-to-conclusion bias is caused by hyperactivity dopaminergic system in brain [10], that is to say, it leads the environmental stimuli to comprehend more conspicuously; thus, the people who suffer from jumping-to-conclusion bias will estimate perceiving stimuli in actually. In other words, the weight of the perceived stimuli is greater than the actual weight.

The beads task is a computerized tool for measuring the jumping-to-conclusion bias in schizophrenia patients with delusion. The results of research conducted using beads task confirmed the bias in patients with schizophrenia compared to other groups $[11,12]$. Moreover, several other research have used this task to investigate the jumping-to-conclusion bias in schizophrenia patients with delusion but have not confirmed the bias yet [13-15].

Several researchers $[12,13]$ have suggested several reasons for the failure to replicate previous results: a) ambiguous instructions in task beads [11], b) fixed stimulus or tasks for subjects [12], and c) the potential limitations of using artificial stimulants in generalizing the results to real-life situations. Task ambiguity in the instruction results in the subjects not realizing the intentions of the examiner and instead utilizing their own interpretation of the task to complete it [11] because of the fact that the examiner offers just a set of beads to the participants, they do not have any opportunities to correct their mistakes; hence, their scores are not a great index for jumping to conclusion bias [12].

It is very important that the jumping to conclusion bias is diagnosed as a cause of delusion in schizophrenic patients, and also for resolving mentioned defects in beads task, the new similarity task was designed. The instructions of similarities task are clear. In this task, a set of images is presented to the participants, and they are tested for their intelligence. Moreover, the test material related to real life situations is less ambiguous and has greater generalizability. Therefore, a major goal of this research is to predict the severity of delusion based on jumping-to-conclusion bias in schizophrenia patients with respect to the similarity task.

\section{Methods}

The current research uses correlation for determining the severity of delusion. The predicted variable is jump- ing-to-conclusion, and the criterion variable is severity delusion. Variables such as gender, age, rate of depression, length of illness, and rate of drug used are the meddler variables.

A total of 60 participants, including hospitalized inpatients and outpatients in the Razi and Ebneina (psychiatry hospitals) in Shiraz, were considered in this study. The participants, with schizophrenia disorders who had experienced delusion with different severity, were asked to perform a similarity task. The participants were diagnosed by their psychiatrists based on the DSM-IV criteria [16]. The delusion severity was assessed with the Scale for Assessment of Positive/Negative Symptom by the researcher. The exclusion criteria were severe substance abuse, any form of documented or suspected brain damage/disease, and an additional Axis I diagnosis.

\section{Similarities task}

The Similarities Task by Saffarian (2010) is a native task to measure the jumping-to-conclusion bias in delusional patients, and the validity and reliability were also reported. The test material consisted of pictures that were similar to each other. The pictures were presented in 17 groups to the participants. The images included the fields and classifications that they dealt with daily, such as home and personal appliances. The way this exam is performed is that in each trial, an image is shown as a particular pattern at the top of the computer screen, and three images are simultaneously shown at the bottom of the screen, then the participant has to realize which one is the same with the picture at the top. Other images have minor differences with template one. Similar images revolve around themselves with five different speeds.

The instruction of task performance is that the examiner ask subjects to guess which image is answer while all three pictures are rotating and click on the most similar image. If the participant guesses the correct image in the first period of rotation (the speed is too fast) he/she will achieve highest score, which is 5. Similarly, if the participant discerns the image in the second one (fast), a score of 4 will be acquired, and in the third period of rotation (normal speed), a score of 3 will be given; in the fourth rotation (slow speed), the score will be 2 ; and finally if he guesses the correct image in the fifth rotation (very slow speed), his score will be 1 . Total score should be in the range of 17 to 85 . In this task, according to the jumping-to-conclusion theory, when trials are presented at the highest speed (the first of rotation), people with jumping-to-conclusion bias are more likely to guess the most similar image. 
In contrast, the people with no jumping-to-conclusion bias guess the most similar image in fourth or fifth period of rotations. In addition, it seems that the participants with jumping-to-conclusion bias because of hasty decisions will make more mistakes in comparison with those who do not have jumping-to-conclusion bias. For this reason, all images circle with five different speeds, including very fast, fast, normal, slow and very slow. The concurrent validity of this task (with beads task) on 45 schizophrenia patients with delusions is equal to -0.76 (because of different ways between task scoring, correlations is negative), and its reliability using Cronbach's Alpha and splitting was 0.95 and 0.87 respectively [17].

Scale for Assessment of Positive Symptom (SAPS) and Scale for Assessment of Negative Symptom (SANS): The SAPS and SANS were administered by an experienced clinician in a semi-structured interview. These instruments together contain 57 items, each covering a specific symptom on a Likert scale from 0 (not present) to 5 (very frequent). The items were clustered into 10 subgroups: five subgroups covered positive symptoms (P1=hallucinations, $\mathrm{P} 2=$ delusions, $\mathrm{P} 3=$ thought disorder, $\mathrm{P} 4=$ bizarre/disorganized behavior, and $\mathrm{P} 5=$ inappropriate affect), and five subgroups covered negative symptoms (N1=restricted affect, N2=alogia: reduced speech. $\mathrm{N} 3=$ avolitionorapathy, N4=a sociality or anhedonia, and $\mathrm{N} 5=$ reduced attention). Lack of insight is not included in the positive symptoms or the negative symptom categories. The total score of the SAPS and SANS is the sum of all responses. The questionnaire has been found with a high inter-rater reliability for the total score, as well as for the positive and negative scales (alpha $=0.86$ ) [18].

\section{Results}

The sample consisted of 60 schizophrenic patients with different delusion severity. Of them, 38.3\%(n=23) were female, and $61.7 \%(n=37)$ were male. The mean age of the participants were 34.13 years $(\mathrm{SD}=11.2$; range $=19-52$ years). The mean length of illness in psychiatry units was 20.45 years $(\mathrm{SD}=10.9)$. Correlations among the jumpingto-conclusion bias and severity of delusion are shown in Table 1. Jumping-to-conclusion bias and severity of delusion were significantly correlated $(\mathrm{P}=0.001)$.

The linear regression analysis (hierarchy method) was conducted to evaluate the assumption of research. In the

Table 1. Correlations between jumping-to-conclusion bias with severity of delusion.

\begin{tabular}{|c|c|}
\hline \multicolumn{2}{|c|}{ Severity of delusion } \\
\hline & $r=0.82 *$ \\
\hline Jumping-to-conclusion bias & \\
\hline
\end{tabular}

${ }^{{ }^{r}}$ =Correlation

Пranian Rehabilitation Journal

Table 2. Result of linear regression analysis (hierarchy method) for predicting delusion variable.

\begin{tabular}{cccccc}
\hline Pattern & $\mathbf{R}$ & $\mathbf{R}^{2}$ & Estimate error & $\mathbf{R}^{2 \text { changes }}$ & P-value \\
\hline 1 & 0.33 & 0.11 & 1.37 & 0.04 & 0.17 \\
2 & 0.78 & 0.61 & 0.91 & 0.57 & 0.001 \\
\hline
\end{tabular}

Table 3. Result of linear regression analysis (hierarchy method) for predicting delusion variable.

IIranian Rehabilitation Journal

\begin{tabular}{|c|c|c|c|c|c|c|c|c|}
\hline \multirow{2}{*}{ Variable } & \multicolumn{4}{|c|}{ First pattern } & \multicolumn{4}{|c|}{ Second pattern } \\
\hline & B & SE & $\mathbf{t}$ & $\mathbf{P}$ & $\mathbf{P}$ & $\mathbf{t}$ & SE & B \\
\hline Age & 0.03 & 0.1 & 1.1 & 0.27 & 0.27 & 1.1 & 0.01 & 0.01 \\
\hline Sex & 0.24 & 0.15 & 1.63 & 0.1 & 0.15 & 1.14 & 0.1 & 0.14 \\
\hline Length of illness & 0.003 & 0.007 & 0.37 & 0.71 & 0.08 & 1.74 & 0.05 & 0.08 \\
\hline Rate of depression & 0.02 & 0.02 & 0.85 & 0.39 & 0.36 & 0.91 & 0.01 & 0.01 \\
\hline \multirow[t]{2}{*}{ Jumping to conclusion bias } & & & & & 0.001 & 8.1 & 0.006 & 0.05 \\
\hline & \multicolumn{4}{|c|}{$R^{2}$} & \multicolumn{4}{|c|}{0.61} \\
\hline
\end{tabular}


first stage, the variables of age, gander, length of illness, and depression rate as control variables were analyzed. The jumping-to-conclusion bias as a predictor variable was analyzed in the second stage. Delusion severity was considered as a dependent variable. The result of the analysis is shown in Table 2 and 3.

The result of the hierarchy regression analysis shows that the effects of the first total variable stage (age, gander, length of illness, depression rate) are not significant. In the second stage, when the jumping-to-conclusion bias was added as a predictor variable, the power of prediction was increased to 0.57 .

\section{Discussion}

The purpose of the study was to answer the hypothesis that the jumping-to-conclusion bias was variable predict delusion. The results showed that this bias can predict delusion. In other words, the severity of delusion will increase when the jumping-to-conclusion bias increases. Some studies have shown that schizophrenia patients with delusion have jumping-to-conclusion bias and this outcome repeats in this research again. Our results showed that this bias can predict delusion severity when jumpingto-conclusion bias is applied to predict the severity of delusion. These findings indicate that jumping-to-conclusion bias can cause delusions. Hyperactivity dopaminergic system may be due to the jumping-to-conclusion bias. That is, it leads the stimulus to perceive explicitly, so the weight of stimulus will be over-estimated and the patient judges about it based on insufficient evidences.

Thus, it can be said that different beliefs are involved in the development or emergence of delusion. In future researches, a model that combines different biases in schizophrenia patients with delusion can have a higher explanatory power for explaining how delusions develop. Furthermore, its results could be used to treat schizophrenia, particularly in cognitive therapy, which is emphasized on decreasing the jumping-to-conclusion bias in patient with delusion. Future research can examine the combination jumping-to-conclusion bias and other biases in the development of delusion.

\section{Conclusion}

The results have shown that jumping-to-conclusion bias can predict the severity of delusion.

\section{Acknowledgments}

We would like to thank the patients who participated in this study. This article was retrieved from Ms. Saffarian's master thesis.

\section{Conflict of Interests}

The authors declared no conflict of interests.

\section{Reference}

[1] Blackwood NJ, Howard RJ, Bentall RP, Murray RM. Cognitive neuropsychiatric models of persecutory delusions. American Journal of Psychiatry. 2014; 158(4):527-39.

[2] Bentall RP, Corcoran R, Howard R, Blackwood N, Kinderman P. Persecutory delusions: a review and theoretical integration. Clinical Psychology Review. 2001; 21(8):1143-192.

[3] Woodward TS, Munz M, Le Clerc C, Lecomte T. Change in delusions is associated with change in "jumping to conclusions". Psychiatry Research. 2009; 170(2):124-27.

[4] Van der Gaag M. A neuropsychiatric model of biological and psychological processes in the remission of delusions and auditory hallucinations. Schizophrenia Bulletin. 2006; 32(1):113-22.

[5] Averbeck BB, Evans S, Chouhan V, Bristow E, Shergill SS Probabilistic learning and inference in schizophrenia. Schizophrenia Research. 2011; 127(1):115-22.

[6] Speechley WJ, Whitman JC, Woodward TS. The contribution of hypersalience to the "jumping to conclusions" bias associated with delusions in schizophrenia. Journal of Psychiatry \& Neuroscience: Journal of Psychiatry Neuroscience. 2010; 35(1):7-17.

[7] Lincoln TM, Ziegler M, Mehl S, Rief W. The jumping to conclusions bias in delusions: specificity and changeability. Journal of Abnormal Psychology. 2010; 119(1):40-49. doi: 10.1037/ a0018118

[8] Veckenstedt R, Randjbar S, Vitzthum F, Hottenrott B, Woodward TS, MoritzS. Incorrigibility, jumping to conclusions, and decision threshold in schizophrenia. Cognitive Neuropsychiatry. 2011; 16(2):174-92. doi: 10.1080/13546805.2010.536084

[9] Moritz S, Veckenstedt R, Randjbar S, Hottenrott B, Woodward T, Eckstaedt FV, et al. Decision making under uncertainty and mood induction: further evidence for liberal acceptance in schizophrenia. Psychological Medicine. 2009; 39(11):1821-829.

[10] Kapur S. Psychosis as a state of aberrant salience: a framework linking biology, phenomenology, and pharmacology in schizophrenia. American Journal of Psychiatry. 2003; 160(1):13-23.

[11] Freeman D. Suspicious minds: the psychology of persecutory delusions. Clinical Psychology Review. 2007; 27(4):42557.

[12] Warman DM, Lysaker PH, Martin JM, Davis L, Haudenschield SL. Jumping to conclusions and the continuum of delusional beliefs. Behaviour Research and Therapy. 2007; 45(6):1255-269 
[13] Garety PA, Freeman D. Cognitive approaches to delusions: a critical review of theories and evidence. British Journal of Clinical Psychology. 1999; 38(2):113-54.

[14] Menon M, Mizrahi R, Kapur S. "Jumping to conclusions" and delusions in psychosis: relationship and response to treatment. Schizophrenia Research. 2008; 98(1):225-31.

[15] Moritz S, Woodward TS. Jumping to conclusions in delusional and non-delusional schizophrenic patients. British Journal of Clinical Psychology. 2005; 44(2):193-207.

[16] American Psychiatric Association. Diagnostic and statistical manual of mental disorders: Primary care version. Washington, D.C.: American Psychiatric Association; 1996.

[17] Saffarian Z. [Predicting the severity of persecutory delusions based on attribusonal bias and jumping to conclusion biases in patients with schizophrenia (Persian)] [Ms Dissertation]. Shiraz: Shiraz University. 2010.

[18] Andreasen N. Scale for the assessment of positive symptoms (SAPS). Iowa: University of Iowa; 1984. 